\title{
Cultura y desarrollo. Perspectivas teóricas para su apropiación por los actores locales
}

\section{Culture and development: theoretical perspectives for local actors}

\section{Axel Polanco Noy*}

\footnotetext{
Docente e investigadora de la Universidad de Camagüey, Cuba. Licenciada en Estudios Socioculturales. Línea de investigación: «La dimensión cultural en la gestión del desarrollo local». Diplomada en Diseño y Gestión de Proyectos Culturales de Desarrollo. Asesora de proyectos en el Departamento de Desarrollo Local del Centro Universitario Municipal (CUM) de Guáimaro.

\·axelita.87@gmail.com http://orcid.org/0000-00031719-5716
}

RECIBIDO: 18.3.2021

\section{Resumen}

La integración de la cultura en la planificación estratégica del desarrollo encuentra una limitación en el insuficiente reconocimiento de sus aportes a pesar del creciente número de estudios sobre este particular. Se aprecia que una escasa preparación de los actores locales constituye un impedimento cardinal para un mayor aprovechamiento de dicho potencial. Por ende, se requiere comprender mejor el vínculo entre ambos términos, en aras de proveerlos de marcos teóricos más amplios para la acción desde el territorio. En el contexto cubano actual esto cobra especial relevancia debido a las transformaciones socioeconómicas que acontecen, por las que los actores locales están llamados a incrementar su actuar en función del desarrollo territorial. Este artículo tiene el propósito de examinar el nexo cultura-desarrollo a partir de diversos postulados teóricos que dan cuenta de esas intersecciones para de esta manera contribuir a su apropiación por los actores en su praxis. Como métodos teóricos se emplearon el histórico-lógico y el análisis-síntesis para el tratamiento de documentos normativos y de la bibliografía relevada.

Palabras clave: cultura, desarrollo económico y social, planificación estratégica. 


\section{Abstract}

The incorporation of culture into strategic planning is limited by the insufficient recognition of its contributions to development, despite the growing number of studies in this field. Local actors' lack of preparation prevents a greater use of this potential. Therefore, it is necessary to better understand the link between both terms to provide them with broader theoretical frameworks for local action. In the current Cuban context, this is especially relevant due to the socioeconomic transformations that are taking place, therefore local actors are called upon to increase their actions in terms of territorial development. This article aims to examine the culture-development nexus from various theoretical standpoints that show these intersections, in order to contribute to their appropriation by the actors in their praxis. Historical-logical and analysis-synthesis methods were used for the treatment of normative documents and the bibliography surveyed.

Keywords: culture, economic and social development, strategic planning.

\section{Introducción}

Mientras se escribe este texto, la humanidad enfrenta una crisis sanitaria global sin precedentes. La pandemia de covid-19 azota con fuerza a las sociedades de cada nación, y sus efectos nocivos sobre todos los ámbitos de la vida social están transformando el mundo conocido. Las diferentes maneras de vivir juntos se han visto obligadas a adaptarse frente a los impactos socioeconómicos de la nueva enfermedad en todos los países. El campo de la cultura y el desarrollo también muestra estrategias de afrontamiento que se ubican en el terreno de lo económico para reconducir el camino hacia el logro de los Objetivos de Desarrollo Sostenible.

En tal sentido, una acción relevante desde las Naciones Unidas es la declaración, apoyada por 81 países, del 2021 como Año Internacional de la Economía Creativa para el Desarrollo Sostenible (Naciones Unidas, 2019). La resolución sitúa nuevamente en el debate internacional el estrecho vínculo entre ambos conceptos, un nexo clave que hay que replantearse necesariamente en una etapa poscovid, en el que Cuba no es la excepción.

En términos generales, la experiencia en el campo del desarrollo ha demostrado que la extrapolación de modelos que se centran en el ámbito económico, sin tomar en cuenta el contexto sociocultural del país donde se aplican, está destinado al fracaso. Un elemento relevante para superar esa realidad es la consideración de la cultura como un factor clave del desarrollo. Aunque los esfuerzos por incorporarla como el cuarto pilar de desarrollo son disímiles, sigue sin aparecer explícitamente en objetivos, programas, planes y estrategias vinculados a los procesos del desarrollo. Así lo demuestran los Objetivos de Desarrollo Sostenible (ODS) y la Agenda 2030, según señala Martinell (2020). 
La bibliografía consultada permite afirmar que esa ausencia de la cultura, parcial o total, obedece, en parte, a la permanencia en los actores del desarrollo de una mentalidad tradicional economicista que carece de una comprensión amplia del nexo culturadesarrollo. Un vínculo que puede asimilarse mejor al entender que «el aporte de la cultura al desarrollo deriva, por una parte, de asumirla como "un modo integral de vida", lo cual marca el horizonte de bienestar y justicia social al que se aspira; y por otra, de aceptarla como un recurso que provee múltiples alternativas en la práctica desarrollista» (Polanco y Caballero, 2020a).

Para ello es preciso explicar y demostrar que la cultura ha estado, implícita o explícitamente, en las concepciones del desarrollo; no obstante, su rol en el cambio social se ha visto condicionado por interpretaciones que reducen o retardan su integración en la gestión del desarrollo a diferentes escalas. Igualmente es necesario argumentar que, debido a su naturaleza tangible e intangible, se demandan conocimientos específicos que permitan aprovecharla mejor en el diseño y la implementación de acciones de cambio, especialmente en la escena local.

El contexto del desarrollo local en Cuba muestra importantes transformaciones en el marco del Plan Nacional de Desarrollo Económico y Social hasta el 2030, que acontecen especialmente en los territorios. Un ámbito que hoy, con más fuerza, se revela estratégico para insertar la dimensión cultural en su doble condición, de conservar la tradición y de generar posibilidades de innovación en acciones no inscritas en los repertorios tradicionales del desarrollo como práctica humana (Espina Prieto, 2010). Su tratamiento, en el periodo que abarca desde el $2011^{1}$ al 2015, se percibe insuficiente en el plano académico, institucional, económico y jurídico (Polanco y Caballero, 2020a, pp. 144-145).

Sin embargo, el reciente Informe Nacional de Desarrollo Humano Cuba 2019 respalda un incremento del interés por la cultura y su papel en el desarrollo local desde el 2016 hasta la actualidad (Programa de las Naciones Unidas para el Desarrollo [PNUD], 2020).

La cultura cada vez más se convierte en elemento clave en las estrategias de desarrollo de los territorios. El potencial creativo, dinamizador e innovador de la cultura favorece una visión y concepción del desarrollo más humanizada, participativa, generadora de conocimientos, de capacidades en las personas, en los grupos y las comunidades. (p. 166)

En esta fecha se celebró el VI Congreso del Partido Comunista de Cuba, en el que se aprobaron los Lineamientos que regulan la política económica y social del partido y la revolución. Ello marca el inicio del proceso conocido como «actualización del modelo económico y social cubano». 
Aunque esto también se refleja en publicaciones académicas que abordan la dimensión cultural (Polanco y Caballero, 2020b; Martínez, 2020, 2018a, 2018b; Isalgué, 2017), los análisis y las experiencias que se exponen no alcanzan para superar el vacío que se constata en la planificación del desarrollo respecto al reconocimiento y el uso de las potencialidades culturales en cada territorio. Lo anterior se advierte en una investigación que se conduce (Polanco y Caballero, 2020a, 2020b), en la que además se revela la desigual e insuficiente preparación que muestran los actores locales para incorporar la dimensión cultural en su gestión.

Al tener en cuenta las transformaciones para dotar a los municipios de mejores capacidades para satisfacer necesidades locales y actuar en función del desarrollo económico y social de la demarcación, así como las oportunidades para el aprovechamiento de la cultura como recurso en la Política para el Impulso al Desarrollo Territorial, propuesta por el Ministerio de Economía y Planificación (MEP) junto con otros actores sociales en el 2019, este trabajo procura proponer un marco teórico que vaya más allá de los aportes de la economía creativa expuestos en informes y artículos académicos (Montero, 2021; de Groot et al., 2020).

Se asume que la visión de los actores locales en torno al nexo cultura-desarrollo constituye un elemento que influye profundamente en el aprovechamiento de este potencial. De ello se deriva el objetivo del presente artículo: examinar dicho vínculo a partir de diferentes postulados teóricos que dan cuenta de sus intersecciones, y de esta manera contribuir a su apropiación en la praxis del desarrollo local en Cuba.

\section{Desarrollo}

Un nuevo marco de acción con relación al desarrollo se inició en septiembre de 2015 para todas las naciones del mundo. La Agenda 2030, aprobada por la Asamblea General de la Organización de Naciones Unidas (ONU), sitúa en el centro de la práctica desarrollista la dignidad y la igualdad de las personas, para lo cual considera esencial la participación de todos los sectores de la sociedad y del Estado (Comisión Económica para América Latina y el Caribe [CEPAL], 2016, p. 5). Materializar los 17 ODS, con sus 169 metas, plantea grandes desafíos prácticos en torno a la interconexión de los pilares del desarrollo sostenible: crecimiento económico, inclusión social y protección del medio ambiente.

Los tres son elementos centrales en el debate respecto al desarrollo y la vida humana, que matizaron la «reemergencia crítica del concepto» (Espina Prieto, 2010) hacia la década del noventa del siglo XX, en la que la sociología, al decir de António et al. (2015), aportó nuevas visiones que el contexto sociohistórico demandaba en razón del agotamiento de los modelos económicos de la modernidad. 
A propósito, vale destacar el esfuerzo realizado por la Comisión Económica para América Latina y el Caribe (CEPAL) y su enfoque sociológico del desarrollo desde el periodo de posguerra. Su labor en aras de modelar objetivos de desarrollo con base en teorías producidas en otros contextos, pero ajustadas a la realidad latinoamericana, merece los créditos respectivos. Con su accionar ha contribuido a orientar sobre la especificidad del continente para evitar la traslación mecánica de las teorías desarrollistas, así como dar cuenta de una estructura social en constante cambio que tiene implicaciones importantes a la hora de fijar metas de progreso social.

Se constata en los estudios cepalinos (Faletto, 2009) el esfuerzo por trazar una perspectiva de desarrollo regional que tiene como médula el tema de la integración. Este enfoque evidenció desde el principio la exigencia de un mejor conocimiento de la identidad sociocultural de la región. Por otro lado, señala la idea de que el deseo de condiciones mejores de vida requiere cierta responsabilidad de acción individual y colectiva, que puede o no existir.

Su inexistencia implica su promoción consciente, lo que puede ser competencia de instituciones no estrictamente económicas, como el sistema educativo, los medios de comunicación, el Estado, los partidos políticos y las organizaciones de la sociedad civil. En este marco se resalta el papel de los factores culturales en el plano subjetivo; ello con relación al fomento de una ética alrededor del esfuerzo colectivo para alcanzar la materialización del bienestar al que se aspira.

De forma general, la reconceptualización del término desde la teoría social en los finales del siglo XX se configura a partir de la propuesta de nuevos paradigmas de desarrollo (Caballero y Yordi, 2009, pp. 40-65) que buscan incluir aspectos ecológicos, sociales y culturales, prácticamente ignorados hasta ese momento. El sólido tratamiento científico de los aspectos sociales en esta etapa se evidencia en los Informes de desarrollo humano (IDH) publicados por el Programa de las Naciones Unidas para el Desarrollo (PNUD) desde 1990.

Temas como participación comunitaria, seguridad humana, atención a la mujer como grupo social vulnerable, relación entre crecimiento económico y desarrollo humano, problemática de la pobreza y aumento de personas excluidas han sido objeto de interés. La prosperidad individual comenzó a ser asociada no solo a la riqueza de que disponen o carecen las personas, sino además a sus expectativas de vida, al acceso a servicios de salud, a la disponibilidad y la calidad de la educación, a las posibilidades y opciones de trabajo, a los deberes, derechos y privilegios políticos y legales de los ciudadanos.

En contraposición al crecimiento económico tan defendido hasta entonces, emerge y cobra fuerza una noción de desarrollo que se interesa en los aspectos subjetivos de la vida humana por considerarlos la meta más importante. En esta línea hay que considerar dos propuestas teóricas: i) las aportaciones sobre calidad de vida y capacidad, de los investigadores Martha Nussbaum y Amartya Sen (1996); ii) el paradigma de 
desarrollo a escala humana, del economista chileno Manfred A. Max-Neef y sus colaboradores A. Elizalde y M. Hopenhayn (2010).

El concepto capacidad, en la visión del desarrollo que postulan Nussbaum y Sen, constituye un aspecto medular para alumbrar un nuevo camino al progreso social.

[...] capacidad entendida como las combinaciones alternativas de funcionamientos entre las cuales una persona puede elegir la vida que tendrá. En este sentido la capacidad de una persona corresponde a la libertad que tiene para llevar una determinada clase de vida, de tal modo que a la calidad de vida se le adiciona el concepto de capacidad que incorpora a la libertad. (Nussbaum y Sen, 1996, pp. 7-23)

La formación de capacidades y el acrecentamiento y el despliegue de las oportunidades de todas las personas son asumidos como propósitos esenciales para el bienestar humano y su calidad de vida; por otro lado, estas categorías nutren teóricamente los $I D H$ realizados anualmente por el PNUD, entre los que se pueden destacar los de 2004 y 2010 por su vínculo con la cultura.

El desarrollo humano es la expansión de las libertades de las personas para llevar una vida prolongada, saludable y creativa; conseguir las metas que consideran valiosas y participar activamente en darle forma al desarrollo de manera equitativa y sostenible en un planeta compartido. Las personas son a la vez beneficiarias y agentes motivadores del desarrollo humano, como individuos y colectivamente. (PNUD, 2010, p. 24)

Respecto a la segunda propuesta, vale señalar su postura crítica ante la falta de integralidad e insuficiencia de las teorías económicas y sociales hasta entonces promulgadas. En esencia, plantea la condición de una teoría de las necesidades humanas para el desarrollo, en virtud de lo cual «el mejor proceso es aquel que permite elevar más la calidad de vida de las personas, lo que dependerá de las posibilidades que tengan los seres humanos de satisfacer adecuadamente sus necesidades humanas fundamentales» (NHF), las que se interrelacionan e interactúan (citado por Caballero y Yordi, 2009, p. 55).

Este paradigma reconoce que las NHF son las mismas en todas las culturas y períodos históricos; «lo que cambia, a través del tiempo y las culturas, es la manera o los medios utilizados para su satisfacción». La elección de la cantidad y calidad de los satisfactores es un aspecto ampliamente influenciado por la cultura en su rol valorativo, al cual se hace referencia más adelante en el texto. No sería errado pensar, entonces, que la dimensión cultural del desarrollo se relacione también con el despliegue de acciones para aumentar las posibilidades de acceso a satisfactores requeridos según contextos sociales específicos. 
El hecho de que las NHF sean reconocidas en su carácter dual — carencia/potencialidad-sirve de base para un accionar más participativo, en la medida en que estas necesidades comprometen, motivan y movilizan. De igual manera, el bienestar social, atendiendo a esta concepción de las necesidades, se relaciona no solo con bienes y servicios, sino también con prácticas sociales, formas de organización, modelos políticos y valores que enriquecen el término. Lo expuesto se convierte en referente teórico para que gobiernos, organizaciones e instituciones puedan materializar la premisa de «poner al ser humano en el centro de los procesos de desarrollo sostenible» (Naciones Unidas, 2012).

Cabe apuntar aquí la conexión entre lo expuesto en el párrafo anterior y los postulados de la corriente antropológica cultura-personalidad, cuyos principales exponentes son Ralph Linton (1893-1953) y Abram Kardiner (1891-1981). Esta corriente profundiza en la manera en que los seres humanos incorporan y viven su cultura. Con relación a los individuos, se cuestiona cómo la cultura los hace actuar y qué conductas provoca. Se parte de la hipótesis de que cada cultura determina cierto estilo de comportamientos comunes al conjunto de individuos que participan en ella. Con respecto al desarrollo, tales postulados pueden enfocarse en el sujeto, en la dualidad actitud/aptitud, y concebirla como herramienta para la acción proactiva. Ello condiciona la elección del camino, de las soluciones, las propuestas de resultados que se procura alcanzar, así como la noción de bienestar (Romero, 2005) que moviliza al individuo y los intereses y valores que unifican la acción.

En el marco reinterpretativo hasta aquí referido, la cultura se revela como una dimensión estratégica e imprescindible del desarrollo, un postulado fuertemente defendido por la Organización de Naciones Unidas para la Educación, la Ciencia y la Cultura (Unesco) desde la década de 1980:

La cultura constituye una dimensión fundamental del proceso de desarrollo y contribuye a fortalecer la independencia, la soberanía y la identidad de las naciones. El crecimiento se ha concebido frecuentemente en términos cuantitativos, sin tomar en cuenta su necesaria dimensión cualitativa [...]. El desarrollo auténtico persigue el bienestar y la satisfacción constante de cada uno y de todos. (Unesco, 1982)

Desde entonces «la dimensión cultural del desarrollo», como categoría conceptual, aparece en escena para dotar dicho proceso de un carácter más humanista, puesto que la cultura «ofrece el contexto, los valores, la subjetividad, las actitudes y las aptitudes sobre las que los procesos de desarrollo han de tener lugar» (Unesco, 1982). Esto se argumenta y demuestra en la sucesión de un grupo importante de acciones entre el último cuarto del siglo XX y lo que va del XXI, que conforman un corpus teórico-práctico imposible ya de ignorar respecto al nexo entre la cultura y el desarrollo (tabla 1). 
Tabla 1. Documentos y acciones en cultura y desarrollo siglo XX y XXI

\begin{tabular}{|c|c|c|}
\hline Documento/acción & Año/contexto & $\begin{array}{l}\text { Actores/agentes del desa- } \\
\text { rrollo involucrados }\end{array}$ \\
\hline $\begin{array}{l}\text { Declaración de México sobre } \\
\text { las Políticas Culturales (Confe- } \\
\text { rencia Mundial) }\end{array}$ & Mundiacult 1982 & Unesco, Estados miembros \\
\hline $\begin{array}{l}\text { Programa de Acción del Dece- } \\
\text { nio para el Desarrollo Cultural }\end{array}$ & 1988-1997 & $\begin{array}{l}\text { ONU, Unesco, Estados } \\
\text { miembros }\end{array}$ \\
\hline $\begin{array}{l}\text { Informe Mundial «Nuestra di- } \\
\text { versidad creativa» }\end{array}$ & 1995 & Unesco \\
\hline $\begin{array}{l}\text { Plan de Acción sobre Políticas } \\
\text { Culturales para el Desarrollo }\end{array}$ & $\begin{array}{l}1998 \\
\text { (Conferencia Interguber- } \\
\text { namental sobre Políticas } \\
\text { Culturales para el Desa- } \\
\text { rrollo) }\end{array}$ & Unesco, Estados miembros \\
\hline $\begin{array}{l}\text { Conferencia «La cultura } \\
\text { cuenta: Recursos financieros y } \\
\text { la economía de la cultura en el } \\
\text { desarrollo sostenible» }\end{array}$ & 1999 & $\begin{array}{l}\text { Unesco, Banco Mundial } \\
\text { (BM) }\end{array}$ \\
\hline $\begin{array}{l}\text { Declaración Universal sobre la } \\
\text { Diversidad Cultural }\end{array}$ & 2001 & Unesco \\
\hline $\begin{array}{l}\text { Convención para la Salvaguar- } \\
\text { dia del Patrimonio Cultural In- } \\
\text { material }\end{array}$ & 2003 & Unesco \\
\hline $\begin{array}{l}\text { Informe «La libertad cultural } \\
\text { en el mundo diverso de hoy» }\end{array}$ & $\begin{array}{l}2004 \\
\text { Informe de desarrollo hu- } \\
\text { mano (IDH) }\end{array}$ & $\begin{array}{l}\text { Programa de Naciones Uni- } \\
\text { das para el Desarrollo } \\
\text { (PNUD) }\end{array}$ \\
\hline Agenda 21 de la Cultura & $\begin{array}{l}2004 \\
\text { (Foro Universal de Cul- } \\
\text { turas) }\end{array}$ & $\begin{array}{l}\text { Ciudades y Gobiernos Loca- } \\
\text { les Unidos (CGLU) }\end{array}$ \\
\hline $\begin{array}{l}\text { Convención sobre la Protec- } \\
\text { ción y Promoción de la Diver- } \\
\text { sidad de Expresiones Cultura- } \\
\text { les }\end{array}$ & 2005 & Unesco \\
\hline 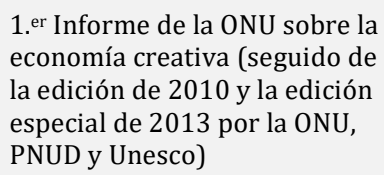 & 2008 & ONU \\
\hline
\end{tabular}




\begin{tabular}{|c|c|c|}
\hline $\begin{array}{l}\text { Informe «Invertir en la diversi- } \\
\text { dad cultural y el diálogo inter- } \\
\text { cultural» }\end{array}$ & $\begin{array}{l}2009 \\
\text { (Informe Mundial de } \\
\text { Cultura) }\end{array}$ & Unesco \\
\hline $\begin{array}{l}\text { Informe «Ciudades, culturas y } \\
\text { desarrollos» }\end{array}$ & $\begin{array}{l}2009 \\
\text { (1. er informe de la } \\
\text { Agenda } 21 \text { de la cultura) }\end{array}$ & CGLU \\
\hline Adopción la A/RES/65/66 & $\begin{array}{l}2010 \\
\left(1 .^{\text {a }} \text { resolución de la }\right. \\
\text { Asamblea General de la } \\
\text { ONU sobre cultura y } \\
\text { desarrollo) }\end{array}$ & ONU \\
\hline $\begin{array}{l}\text { Informe sobre cultura y desa- } \\
\text { rrollo A/66/187 }\end{array}$ & $\begin{array}{l}2011 \\
\text { (Adopción de la 2. a reso- } \\
\text { lución de la Asamblea } \\
\text { General de la ONU sobre } \\
\text { cultura y desarrollo } \\
\text { A/RES/66/208) }\end{array}$ & Unesco, ONU \\
\hline $\begin{array}{l}\text { Presentación de la Iniciativa F- } \\
\text { ODM «Hacer de la cultura un } \\
\text { motor para el desarrollo» }\end{array}$ & 2011 & Unesco \\
\hline $\begin{array}{l}\text { Implementación de indicado- } \\
\text { res Unesco de cultura para el } \\
\text { desarrollo sostenible }\end{array}$ & 2014 & Unesco, Estados miembros \\
\hline Adopción de A/RES/69/230 & $\begin{array}{l}2015 \\
\left(4 .^{\mathrm{a}} \text { resolución de la ONU }\right. \\
\text { sobre cultura y desarrollo) }\end{array}$ & \\
\hline $\begin{array}{l}\text { Campaña mundial «El futuro } \\
\text { que queremos» por la inclu- } \\
\text { sión de la cultura en los ODS } \\
2015\end{array}$ & $\begin{array}{l}2015 \\
\text { (www.cul- } \\
\text { ture2015goal.net ) }\end{array}$ & $\begin{array}{l}\text { Federación Internacional } \\
\text { de Consejos de Artes y } \\
\text { Agencias Culturales (FI- } \\
\text { CAAC), CGLU (comisión de } \\
\text { cultura), Federación Inter- } \\
\text { nacional de Coaliciones por } \\
\text { la Diversidad Cultural } \\
\text { (FICDC), Culture Action Eu- } \\
\text { rope, Arterial Network, } \\
\text { Consejo Internacional de la } \\
\text { Música (CIM), Consejo In- } \\
\text { ternacional de Monumen- } \\
\text { tos y Sitios (ICOMOS) }\end{array}$ \\
\hline $\begin{array}{l}\text { Informe Mundial de la Unesco } \\
\text { «Cultura: Futuro urbano» }\end{array}$ & 2016 & Unesco \\
\hline $\begin{array}{l}\text { Informe «Diálogos post-2015 } \\
\text { sobre cultura y desarrollo» }\end{array}$ & 2016 & $\begin{array}{l}\text { Unesco, PNUD y el Fondo } \\
\text { de Población de las Nacio- } \\
\text { nes Unidas (UNFPA) }\end{array}$ \\
\hline
\end{tabular}




\begin{tabular}{|c|c|c|}
\hline $\begin{array}{l}2 .^{\circ} \text { Informe Mundial de la } \\
\text { Unesco «Repensar las políticas } \\
\text { culturales» }\end{array}$ & 2017 & Unesco \\
\hline $\begin{array}{l}\text { La cultura para la Agenda } \\
2030\end{array}$ & $\begin{array}{l}2018 \\
\text { (folleto que divulga las } \\
\text { mejores prácticas de la } \\
\text { Unesco en relación con } \\
\text { los ODS) }\end{array}$ & Unesco \\
\hline
\end{tabular}

Fuente: Elaboración propia, 2019.

Se puede comprobar, con base en las acciones referidas, que el esfuerzo para demostrar que «solo puede asegurarse un desarrollo equilibrado mediante la integración de los factores culturales en las estrategias para alcanzarlo» (Unesco, 1982) ha sido arduo e inagotable. Si bien no parecen existir impedimentos para integrar la cultura en los procesos de desarrollo a diversas escalas y su respectiva planeación, lo cierto es que, en los ODS, el principal marco de acción para el progreso social a escala internacional no se incluye explícitamente. No obstante, el punto 36 del texto ciertamente alude a las culturas y a la diversidad cultural, cuya contribución se reconoce:

Nos comprometemos a fomentar el entendimiento entre distintas culturas, la tolerancia, el respeto mutuo y los valores éticos de la ciudadanía mundial y la responsabilidad compartida. Reconocemos la diversidad natural y cultural del mundo, y también que todas las culturas y civilizaciones puedan contribuir al desarrollo sostenible y desempeñen un papel crucial en su facilitación. (CEPAL, 2016)

Martinell (2015) críticamente apunta algunos elementos que explican este relativo olvido. Entre ellos señala «la influencia de cierta mentalidad tradicional de no aceptar o no entender la cultura como un factor imprescindible del desarrollo en el siglo XXI». Este pensamiento tiene su base en el conflicto entre este término y los conceptos de necesidades básicas, pobreza, hambre, etcétera, en el que se maneja una constante confrontación de prioridades que se agudiza con «posiciones paternalistas y asistencialistas que consideran las necesidades culturales como algo prescindible o un lujo en situaciones de pobreza».

Tal concepción es objetada por Sen (2004), quien alega que «los economistas pueden, con resultados ventajosos, poner más atención a la influencia de la cultura en los asuntos económicos y sociales». De manera generalizada existe la creencia de que las diferencias entre países ricos y pobres están determinadas por condiciones económicas. El economista respalda que existen otros aspectos de naturaleza cultural que también influyen con fuerza en un mayor o menor progreso de los países (pp. 50-52). 
A pesar de ello, se mantiene vigente una resistencia, desde la visión más tradicional del desarrollo económico, a incorporar los aportes resultantes de estudios en el sector cultural sobre su contribución al producto interno bruto (PIB) y a otros aspectos relacionados con el desarrollo humano, la sociabilidad, la convivencia y la cohesión social, en marcos de acción específicos. Para profundizar en las referidas investigaciones se pueden consultar Benavente y Grazzi, 2018; Kulesz, 2017; Unesco, 2016; Instituto Nacional de Estadística y Censos [INDEC], 2017; Organización de Estados Iberoamericanos [OEI], 2016.

Ello confirma el hecho de que las concepciones teóricas del desarrollo «no lo han situado como un factor determinante e imprescindible, y cuando se ha incorporado ha sido desde una perspectiva muy generalista» (Martinell, 2012, p. 2). Dada la influencia de la cultura en la vida humana (Sen, 2004), es relevante comprender las diferentes formas y naturaleza de esa relación, así como sus implicaciones para la implementación de políticas de desarrollo. De ahí que una mejor comprensión del nexo cultura-desarrollo requiera un análisis más profundo sobre las maneras de «tomar en cuenta la cultura» en los procesos de este tipo y las estrategias para lograrlo.

Aportaciones de autores como Martinell (2015, 2012, 2010), Romero (2005), Sen (2004; 2003), Appadurai (2004), Santillán (2003) y Radl (2000) en torno a la teorización de los términos cultura y desarrollo sugieren:

1. La cultura ha estado, implícita o explícitamente, en las concepciones del desarrollo; no obstante, su rol en el cambio social se ha visto condicionado por interpretaciones sobre su definición que limitan o retardan su integración al proceso.

2. El aporte de la cultura al desarrollo proviene, por una parte, de asumirla como «un modo integral de vida», lo cual marca el horizonte de bienestar y justicia social al que se aspira, $\mathrm{y}$, por otra, de reconocerla como un recurso que provee múltiples alternativas en la gestión del desarrollo a diferentes escalas.

Si, como señala Carranza (2000, p. 66), la cultura «expresa las creencias, las aspiraciones, el conocimiento y las maneras de hacer las cosas de un determinado pueblo», entonces el «progreso económico debe corresponder y potenciar esa realidad específica y no plantearse en conflicto con ella». No obstante, la presencia de la cultura dependerá de qué visión de desarrollo se tenga.

Respecto a la evolución histórica del concepto de desarrollo, pueden consultarse autores como Pérez (2010) y Espina Prieto (2010, pp. 63-69); esta última establece cinco momentos. 
Tabla 2. Síntesis de la evolución histórica del concepto de desarrollo

\begin{tabular}{|c|c|c|}
\hline Momento & Período & Noción de desarrollo \\
\hline I & Siglo XIV-1. a mitad del siglo XIX & Generación \\
\hline II & 2. ${ }^{\text {a }}$ mitad del siglo XIX-1945 & $\begin{array}{l}\text { Universalización } \\
\text { Se establece que desa- } \\
\text { rrollo= modernización }\end{array}$ \\
\hline III & Década del cuarenta-inicios de los setenta & $\begin{array}{l}\text { «Encantamiento del } \\
\text { desarrollo» }\end{array}$ \\
\hline IV & $\begin{array}{l}\text { 2. a mitad de los setenta, década del ochenta e } \\
\text { inicios de los noventa }\end{array}$ & $\begin{array}{l}\text { Crisis del discurso desa- } \\
\text { rrollista }\end{array}$ \\
\hline V & Desde los noventa hasta la actualidad & $\begin{array}{l}\text { Reemergencia crítica del } \\
\text { concepto de desarrollo }\end{array}$ \\
\hline
\end{tabular}

Fuente: Elaboración propia a partir del artículo de Espina (2010).

En el transcurrir de estos momentos, se afianzan dos corrientes principales en torno al concepto, que Sen (2003) denomina como «noción opulenta», profundamente influida por el crecimiento económico y sus valores subyacentes, y «noción de desarrollo de la libertad real», asociada a los valores de los involucrados en el proceso y, por tanto, con un enfoque de progreso social y económico.

Como argumentos que sustentan la segunda noción de desarrollo, dicho autor declara que la cultura tiene un papel fundacional, puesto que el proceso se orienta al enriquecimiento y la expansión de las capacidades humanas — referidas estas a combinaciones de funcionamientos o gamas de opciones que deben tener las personas para decidir la clase de vida que aspiran a tener-, en correspondencia con las valoraciones que los individuos hacen en relación estrecha con su modo de vida.

Por ende, «la pobreza de una vida no reside en la condición de pobreza material en que vive una persona, sino en la falta de una oportunidad real dada tanto por limitaciones sociales como por circunstancias personales para elegir otra forma de vida» (Sen, 2003, p. 70). En esa cuerda, el autor argumenta tres maneras en que la cultura cuenta para el desarrollo, a las que designa roles: constituyente, valorativo e instrumental.

El rol constituyente alude a que el desarrollo, en su sentido más amplio, incluye el desarrollo cultural, un componente básico e inseparable de dicho proceso. En este sentido se destaca la importancia de proporcionar, en forma creciente, oportunidades para que las personas cultiven su creatividad, no solo en el ámbito de las artes y las letras (el más atendido por las políticas culturales) sino en otros espacios vitales de la sociedad que hagan legítimo el derecho humano de participar en la vida cultural de su nación, o 
sea, «gozar de las artes y participar del progreso científico y de los beneficios que de él resulten» (Unesco, 1982).

El papel valorativo refuerza la idea de que los aspectos objetivos y subjetivos de la vida cotidiana que las personas valoran se hallan influidos por la cultura. Esto significa que sus aspiraciones, sea cual sea su procedencia social, tienen que ver con su modo de vida. Por tanto, valorarán bienes y servicios de acuerdo a la cultura que viven y recrean cada día de su existencia, lo que, a su vez, orientará y motivará su acción individual y colectiva para alcanzar el objeto de sus aspiraciones.

Para el sociólogo Peter Worsley (citado por Austin, 2002, pp. 86-87), los valores «son concepciones generales de "lo que es bueno", ideas acerca de la clase de fines que la gente debería buscar y/o perseguir a lo largo de sus vidas y a través de las muchas actividades en que se comprometen». El término valores siempre determina comparación de utilidad e inutilidad de algo; implica en cuánto se estima algo, lo que se halla en relación con normas, símbolos, costumbres, cultura material, etcétera, de una sociedad específica.

Respecto al papel instrumental de la cultura, Sen (2003) refiere que está presente en ambas nociones de desarrollo, en uno con intereses de crecimiento puramente económico y en otro con un desempeño fundacional; o sea, como un factor del progreso humano en sentido amplio. Tal planteamiento revela la función de recurso que se le atribuye a la cultura (Yúdice, 2002; Abello et al., 2010), que desde dicha perspectiva aporta tanto a la dimensión económica como a otros aspectos relativos a la calidad de vida, sobre todo al tomar como referente el paradigma de desarrollo a escala humana.

Por ello, se debe poner atención en las maneras en que influye tanto en asuntos económicos como sociales, para así poder considerarlas en relación con los desafíos prácticos de justicia y equidad del desarrollo y con la valoración de la necesidad de estrategias económicas armoniosas con la identidad local y el medio ambiente. Sen (2004, pp. 39-43) identifica siete manifestaciones del nexo cultura-desarrollo que demuestran esas maneras de influir.

En primer lugar alude nuevamente a los papeles constitutivo y valorativo, cuando expresa: «El fortalecimiento del bienestar incluye el enriquecimiento de la vida humana a través de la literatura, la música, las bellas artes y otras formas de expresión y prácticas culturales que tenemos razón para valorar», y luego: «Los factores culturales tienen un papel central en la formación de valores; estos a su vez pueden influir en la identificación de nuestros fines y en el reconocimiento de instrumentos practicables y aceptables para alcanzar dichos fines».

Refiere además a los objetos y actividades culturales económicamente remunerativas y su especial vínculo con el turismo; a la influencia de los factores culturales en la responsabilidad social, la administración dinámica, las iniciativas emprendedo- 
ras, el comportamiento amigable con el entorno, así como a «toda una gama de aspectos del comportamiento humano que pueden ser cruciales para el éxito económico». De igual forma, menciona su influencia en el establecimiento de alianzas y promoción de la solidaridad en el cuidado de bienes comunes y la protección a los más vulnerables.

Por todo lo expuesto, se asume como desarrollo «un proceso creador, participativo, de carácter consciente que se manifiesta a través de la actividad humana, condicionado por el contexto histórico-social concreto y los diversos móviles ideológicos que conllevan a determinados resultados de autotransformación del entorno y sus individuos, en correspondencia con las necesidades humanas fundamentales y las capacidades reales de los sistemas para contribuir al bienestar y la calidad de vida humanos» (Caballero y Yordi, 2009, p. 36).

Dicha definición permite valorar y constatar las conexiones del proceso con la cultura, puesto que indica rasgos que dan fe de ello: la contribución al bienestar y a la calidad de vida, lo que coincide con situar al ser humano como principal actor y beneficiario al emplear su creatividad y potenciar su participación; el carácter objetivo/subjetivo de la transformación colectiva e individual, condicionada por el contexto y los ideales, y estrechamente relacionada con necesidades sentidas y recursos disponibles.

Como se ha dicho, las definiciones de cultura y su uso en las ciencias sociales ( $\mathrm{Cu}$ che, 2002) han influido en su integración al desarrollo social. Hay que señalar como un posible origen de las posiciones retardantes el empleo indistinto de los conceptos de civilización y cultura hasta la década del sesenta. Hacia el siglo XIX e inicios del XX, cobró fuerza la sociología científica. En este contexto se privilegió el término civilización para pensar la pluralidad cultural en las sociedades humanas.

La postura francesa asume cultura como algo propio del ser humano, saberes acumulados, educación, instrucción en el plano individual; evoca de este modo el progreso individual, mientras que relaciona civilización con el progreso colectivo. Por su parte, la sociología alemana afianza el concepto de civilización al asumirlo como un proceso de mejoramiento de las instituciones, de la legislación, de la educación. Esta última implica una posición excluyente, homogeneizadora, etnocentrista, ligada a una concepción progresista de la historia (Cuche, 2002) que ignora la diversidad cultural.

La civilización es un movimiento no terminado... que afecta a toda la sociedad, comenzando por el Estado, que debe liberarse de todo lo que aún es no razonable en su funcionamiento [...] puede y debe extenderse a todos los pueblos que componen la humanidad [...] todos los pueblos [más civilizados o más salvajes] tienen la vocación de entrar en el mismo movimiento de civilización. (Cuche, 2002, p. 12) 
Durkheim (1858-1917), en sus contribuciones al tema, se aparta del esquema unilineal de evolución común de todas las sociedades. Desarrolló una sociología con orientación antropológica que comprende lo social en todas sus dimensiones y aspectos, incluida la dimensión cultural, a través de todas las formas de sociedades, y apuntó el carácter implícito de cultura en el concepto de civilización:

Y hay que entender por civilización un conjunto de fenómenos sociales que no están vinculados con un organismo social particular; estos se extienden por áreas que superan el territorio nacional o bien se desarrollan en períodos temporales que superan la historia de una sola sociedad. (Durkheim y Mauss, 1909-1912, p. 47)

De esto se entiende que «nada autoriza a creer que los diferentes tipos de pueblos van todos en el mismo sentido, hay algunos que siguen los caminos más diversos. El desarrollo humano debe imaginarse no como una línea en la que las sociedades se dispondrían unas detrás de las otras, como si las más avanzadas no fuesen más que la continuación de las más rudimentarias, sino como un árbol con ramas múltiples y divergentes [...]» (Durkheim citado por Cuche, 2002, p. 31).

Cabe suponer que los aportes de sociólogos y antropólogos, a partir de enfoques diferentes para comprender aspectos conflictivos de la sociedad, hayan situado en el debate académico las implicaciones prácticas de la cultura en las relaciones humanas y, en consecuencia, hacia la segunda mitad del siglo XX se pusiera mayor atención a su vínculo con el desarrollo, tal como lo demuestran los documentos publicados por el sistema de instituciones de las Naciones Unidas antes aludidos, que denotan un consenso internacional con relación al nexo cultura-desarrollo.

Los argumentos anteriores patentizan la necesidad de partir de una definición de cultura que devele las posibilidades de su integración en la gestión del desarrollo para aprovechar con ello todo su potencial (Olazábal et al., 2021). La concepción socioantropológica difundida por la Unesco (1982) la asume como «el conjunto de rasgos distintivos, espirituales y materiales, intelectuales y afectivos que caracterizan a una sociedad o grupo social. Ello engloba, además de las artes y las letras, los modos de vida, los derechos fundamentales del ser humano, los sistemas de valores, las tradiciones y las creencias».

Se reconoce el aporte de esta concepción en la promoción de nuevos marcos de acción para las políticas culturales y su necesaria centralidad en el desarrollo (Unesco, 1998) al afirmar que, «siendo uno de los principales componentes de una política de desarrollo endógena y duradera, debe ser implementada en coordinación con otras áreas de la sociedad en un enfoque integrado. Toda política para el desarrollo debe ser profundamente sensible a la cultura misma». 
El investigador latinoamericano Ricardo Santillán (2003) considera la cultura como el cultivo de:

[...] una forma integral de vida creada histórica y socialmente por una comunidad a partir de su particular manera de resolver — desde lo físico, emocional y mental- las relaciones que mantiene con la naturaleza, consigo misma, con otras comunidades y con lo que considera «trascendente», con el propósito de dar continuidad, plenitud y sentido a la totalidad de su existencia.

Abello et al. (2010, p. 75) apuntan que son pocos los conceptos en las ciencias sociales que han sido objeto de tan profunda actividad reinterpretativa en las últimas décadas. De ahí que las «teorizaciones sobre sus posibles intersecciones contengan nuevas propuestas que evidencian relaciones en doble vía y a través de diversos canales». Un análisis detenido sobre la aportación de Santillán Güemes revela esas conexiones entre ambos términos y confirma su compatibilidad y el carácter definitorio mutuo. Esto último lo argumentan Abello et al. (pp. 78-88) desde tres tipos fundamentales de relación: «la cultura como un medio para el desarrollo, la cultura como contexto del desarrollo y la cultura como fin del desarrollo».

Respecto al desarrollo local, las relaciones mencionadas pueden ser observadas por los actores con nuevos enfoques sobre funciones de la cultura que deberán considerarse en las estrategias que se diseñen. De acuerdo con Martinell (2010, p. 8), algunas de ellas son: impactos directos en el desarrollo socioeconómico y la creación de renta disponible y bienestar, como vía para crear un clima cultural basado en la confianza mutua, la libertad cultural y las relaciones de respeto a la diversidad expresiva; su función de capital humano como medio de obtener poder y reconocimiento social y político; su incidencia en el aumento de las oportunidades sociales de las personas al influir en su educación, el empleo y uso del tiempo libre; su rol imprescindible en una perspectiva integral del desarrollo sostenible.

En consonancia con este autor, el aporte de la cultura al desarrollo se puede resumir en cuatro grandes dimensiones (Martinell, 2010, p. 10-17): 1) la cultura como un conjunto de valores simbólicos, creencias y como expresión de identidades; 2) impactos intangibles de la cultura al desarrollo; 3) la cultura como dimensión e impacto socioeconómico, y 4) la cultura como plusvalía al desarrollo de otros sectores o impactos indirectos. Todo ello presenta fuertes conexiones con los postulados teóricos de los autores examinados aquí. 


\section{Conclusiones}

A manera de resumen de lo expuesto, es importante destacar la necesidad de comprensión del nexo cultura-desarrollo por los actores locales, para así potenciar su posterior incorporación en la planificación y la implementación de estrategias y proyectos de desarrollo en los territorios donde actúan. Entre los elementos que no deben faltar para comprender mejor esa relación, y por consiguiente su implicación práctica, se incluyen:

- los paradigmas de desarrollo que implican los factores culturales en sus fundamentos teóricos;

- definiciones y usos sociales de cultura que develan su rol en el proceso y sus aportaciones;

- nuevos marcos de acción de la política cultural, que permitan su integración desde un enfoque integral del desarrollo sostenible;

- otras categorías, que fundamentan visiones amplias de la interrelación entre desarrollo y cultura, que deben ser consideradas en la planificación del proceso: nociones locales de bienestar, calidad de vida, necesidades fundamentales humanas, capacidades, aspiraciones, valores.

\section{Referencias bibliográficas}

Abello, A., Aleán, A., y Berman, E. (2010). Cultura y desarrollo: Intersecciones vigentes desde una reflexión conceptual reflexiva. En A. Martinell (coord.), Cultura y desarrollo. Un compromiso para la libertad y el bienestar (pp. 75-90). Madrid: Siglo XXI.

António, D., Mesa, J., Martínez, A., y Expósito, E. (2015). El enfoque microsociológico en los estudios del desarrollo local. Santiago, (137), 625-644.

Appadurai, A. (2004). The capacity to aspire: Culture and the terms of recognition. En V. Rao y M. Walton (ed.), Culture and public action (pp. 59-84). Stanford, California: Stanford University Press.

Austin, T. (2002). Para una teoría de la cultura. En T. Austin, Fundamentos socioculturales de la educación. Victoria, Chile: Universidad Arturo Prat.

Benavente, J. M., y Grazzi, M. (2018). Impulsando la economía naranja en América Latina y el Caribe. Montevideo: Unesco.

Caballero, M. T., y Yordi, M. (2009). EL desarrollo social y el trabajo comunitario: Teoría, metodología y prácticas cubanas. Camaguey: Ácana.

Carranza, J. (2000). Cultura y desarrollo: Algunas consideraciones para el debate. Cultura y Desarrollo, 63-78. 
Comisión Económica para América Latina y el Caribe. (2016). Agenda 2030 y los Objetivos de Desarrollo Sostenible. Santiago: Naciones Unidas.

Cuche, D. (2002). La noción de cultura en las ciencias sociales. Buenos Aires: Nueva Visión.

de Groot, O., Dini, M., Gligo, N., Peralta, L., y Rovira, S. (2020). Economía creativa en la revolución digital: La acción para fortalecer la cadena regional de animación digital en países mesoamericanos. Santiago: CEPAL.

Durkheim, E., y Mauss, M. (1909-1912). Note sur la notion de civilisation. L'Année Sociologique, 12, 46-50.

Espina Prieto, M. (2010). Desarrollo, desigualdad y políticas sociales. La Habana: Acuario.

Faletto, E. (2009). Dimensiones sociales, políticas y culturales del desarrollo. Bogotá: Siglo del Hombre.

Instituto Nacional de Estadística y Censos. (2017). Cuenta satélite de la cultura: Metodología de estimación del valor agregado bruto y comercio exterior cultural. Buenos Aires: INDEC.

Isalgué, Y. (2017, julio). Programa de desarrollo local y comunitario desde la cultura. Revista Caribeña de Ciencias Sociales. Recuperado de http://www.eumed.net/ rev/caribe/2017/07/desarrollo-local-guantanamo.html.

Kulesz, O. (2017). La cultura en el entorno digital: Evaluar el impacto en América Latina y en España. París: Unesco.

Martinell, A. (2010). Cultura y desarrollo: Un compromiso para la libertad y el bienestar. Madrid: Siglo XXI.

Martinell, A. (2012). Las relaciones entre cultura y desarrollo en el contexto actual. Recuperado de https://www.campuseuroamericano.org/pdf/es/ES_Relaciones_Cultura_Desarrollo_A_Martinell.pdf.

Martinell, A. (2015). ¿Por qué los ODS no incorporan la cultura? Recuperado de http://alfonsmartinell.com/por-que-los-ods-no-incorporan-la-cultura.

Martinell, A. (2020). Por qué los Objetivos de Desarrollo Sostenible no incorporan la cultura. En A. Martinell (dir.), Cultura y desarrollo sostenible: Aportaciones para el debate sobre la dimensión cultural de la Agenda 2030 (pp. 8-15). Madrid: REDS.

Martínez, D. (2018a). La dimensión cultural del desarrollo: Su concepción en el trabajo comunitario. Estudios del Desarrollo Social: Cuba y América Latina, 14(1), 287-306.

Martínez, D. (2018b). Planear el desarrollo desde su dimensión cultural: Un reto post2015. Salud y Administración, 5(14), 43-58.

Martínez, D. (2020). Las políticas culturales post-2015: La experiencia de Cuba. Estudios sobre las Culturas Contemporáneas, 27(52), 37-70.

Max-Neef, M., Elizalde, A., y Hopenhayn, M. (2010). Desarrollo a escala humana: Una opción para el futuro. Madrid: Biblioteca $\mathrm{CF}+\mathrm{S}$. 
Montero, A. (2021). Economías creativas en centros históricos: La Habana Vieja. Bitácora Urbana Territorial, 31(2), 189-202. D0I:10.15446/bitacora.v31n2.86110.

Naciones Unidas. (2012). Resolución aprobada por la Asamblea General el 27 de julio de 2012: El futuro que queremos. Recuperado de https://undocs.org/pdf?symbol=es/ A/RES/66/288.

Naciones Unidas. (2019). Año internacional de la economía creativa. Recuperado de https://undocs.org/sp/A/RES/74/198.

Nussbaum, M., y Sen, A. (1996). La calidad de vida. México: Fondo de Cultura Económica.

Olazábal, M., Rodríguez, V., y González, R. (2021). La identidad cultural como recurso local y su integración a la gestión del desarrollo territorial. Retos de la Dirección, 15(1), 27-60.

Organización de Estados Iberoamericanos. (2016). Estudio comparativo de cultura y desarrollo en Iberoamérica. Madrid: Fundación Santillana.

Pérez, A. (2010). Desarrollo local: Estudio sobre las condiciones del gobierno para generar desarrollo local en el municipio de Manicaragua, provincia de Villa Clara. (Tesis doctoral). Universidad de la Habana, Santa Clara.

Polanco, A., y Caballero, M. (2020a). La gestión de la cultura en el desarrollo local: Aproximaciones desde el fortalecimiento de capacidades. Cuadernos del CLAEH, 39(111), 135-151.

Polanco, A., y Caballero, M. (2020b). Fortalecimiento de capacidades de los actores locales para una gestión del desarrollo local desde la cultura. Retos de la Dirección, 14(1), 45-67.

Programa de las Naciones Unidas para el Desarrollo. (2010). La verdadera riqueza de las naciones: Caminos al desarrollo humano. Nueva York: PNUD.

Programa de las Naciones Unidas para el Desarrollo. (2020). Informe nacional de desarrollo humano. Cuba 2019. La Habana: Selvi.

Radl, A. (2000). La dimensión cultural, base para el desarrollo de América Latina y el Caribe: Desde la solidaridad a la integración. Buenos Aires: BID.

Romero, R. (2005). ¿Cultura y desarrollo? ¿Desarrollo y cultura? Lima: PNUD.

Santillán, R. (2003). El campo de la cultura. En H. Olmos y R. Santillán, Educar en cultura: Ensayos para una gestión integrada. Buenos Aires: Ciccus.

Sen, A. (2003). La cultura como base del desarrollo contemporáneo. Cultura y Desarrollo, (2), 69-73.

Sen, A. (2004). How does culture matter? En V. Rao, y M. Walton (ed.), Culture and public action (pp. 37-58). Stanford, California: Stanford University Press.

Unesco. (1982). Declaración de México sobre las políticas culturales. Recuperado de http://biblioteca.udgvirtual.udg.mx/jspui/handle/123456789/2261. 
Unesco. (1998). Conferencia intergubernamental sobre políticas culturales para el desarrollo. Estocolmo. Recuperado de https://unesdoc.unesco.org/ark:/48223/ pf0000113935_spa.

Unesco. (2016). Diálogos post-2015 en cultura y desarrollo. México: Unesco.

Yúdice, G. (2002). El recurso de la cultura. Barcelona: Gedisa. 